\title{
Tumour metabolic activity measured by fluorodeoxyglucose positron emission tomography for radiotherapy planning as a prognostic factor for locally advanced non-small cell lung cancer
}

\author{
Masarykova $\mathrm{A}^{1}$, Scepanovic $\mathrm{D}^{1}$, Povinec $\mathrm{P}^{2}$, Bires $\mathrm{P}^{1}$, Lederleitner $\mathrm{D}^{1}$, Pobijakova $\mathrm{M}^{1}$ \\ Department of Radiation Oncology, National Cancer Institute, Bratislava, Slovakia. \\ andrea.masarykova@gmail.com
}

\begin{abstract}
OBJECTIVES: To analyse whether the maximum standardized uptake value of the positron emission tomography/computed tomography for radiotherapy planning was useful as a prognostic factor for tumour response and survival of patients with locally advanced non-small cell lung cancer.

BACKGROUND: Increased 18F-fluoro-2-deoxyglucose uptake by lung cancer cells, measured as the maximum standardized uptake value, has been reported to predict the biologic aggressiveness of both early and advanced non-small cell lung cancer.

METHODS: A prospective study was performed in 61 consecutive patients with unresectable stage IA-IIIB of non-small cell lung cancer. The mean age was 65 years. Seventy five percent of patients in the entire group received an induction chemotherapy. The mean dose of radiotherapy was $61 \mathrm{~Gy}$. All patients underwent $18 \mathrm{~F}-$ fluoro-2-deoxy-D-glucose positron emission tomography/computed tomography for radiotherapy planning.

RESULTS: Thirty six percent of the patients experienced a complete response and $20 \%$ had a partial tumour response. Forty four percent of the patients suffered from a progressive disease. The maximum standardized uptake value of the primary tumour more than 11.4 was correlated with a worse tumour response $(p=0.0001)$ and a shorter survival of our patients $(p=0.0109)$.

CONCLUSION: We found a correlation between the maximum standardized uptake value and the patient prognosis and lung cancer aggressiveness (Tab. 3, Fig. 5, Ref. 18). Text in PDF www.elis.sk.

KEY WORDS: carcinoma, non-small-cell lung, induction chemotherapy, radiotherapy, positron-emission tomography, prognostic factors.
\end{abstract}

\section{Introduction}

A prognostic factor is generally defined as a factor, measured before treatment, that has an impact on a patient's outcome "independently" of received treatment or of the general class of treatment (1). With the exception of stage and performance status, no prognostic factors have been definitively established in lung cancer (2). Positron emission tomography/computed tomography (PET/CT) imaging of cancer with combined positron emission tomography (PET) and x-ray computerized tomography (CT) scanners become a standard component of diagnosis and staging in oncology. In addition to cancer detection and staging, PET/CT imaging is becoming more important as a quantitative monitor of individual response to therapy (3). Increased fluorodeoxyglucose

${ }^{1}$ Department of Radiation Oncology, National Cancer Institute, Bratislava, Slovakia, and ${ }^{2}$ PET Centrum BIONT, a.s., Bratislava, Slovakia

Address for correspondence: A. Masarykova, MD, Department of Radiation Oncology, National Cancer Institute, Klenova 1, SK-810 50 Bratislava, Slovakia.

Phone: +421.2 .59378708$
(FDG) uptake by lung cancer cells, measured as the maximum standardized uptake value (SUVmax), has been reported to predict the biologic aggressiveness of both early and advanced nonsmall cell lung cancer (NSCLC) $(4,5)$. The association between higher SUVmax in 18F-fluoro-2-deoxyglucose (18F-FDG) PET/ $\mathrm{CT}$ and a poor prognosis or treatment response in cancer patients has been reported in several prior studies $(6,7,8)$. However, the reliability of SUVmax remains controversial. Some studies found no correlation between SUVmax and patient prognosis or lung cancer aggressiveness (9). Reasonable explanations for this include the following: 1) SUVmax reportedly varies among PET scanners; 2) uncontrolled factors such as: glucose level, duration of the uptake period, body weight, body composition, and recovery coefficient introduce considerable variations in SUVmax; 3) different acquisition and image reconstruction parameters also affect SUVmax $(10,11)$.

\section{Methods}

A prospective study was performed in 61 consecutive patients with unresectable stage IA-IIIB of NSCLC with/without an induc- 

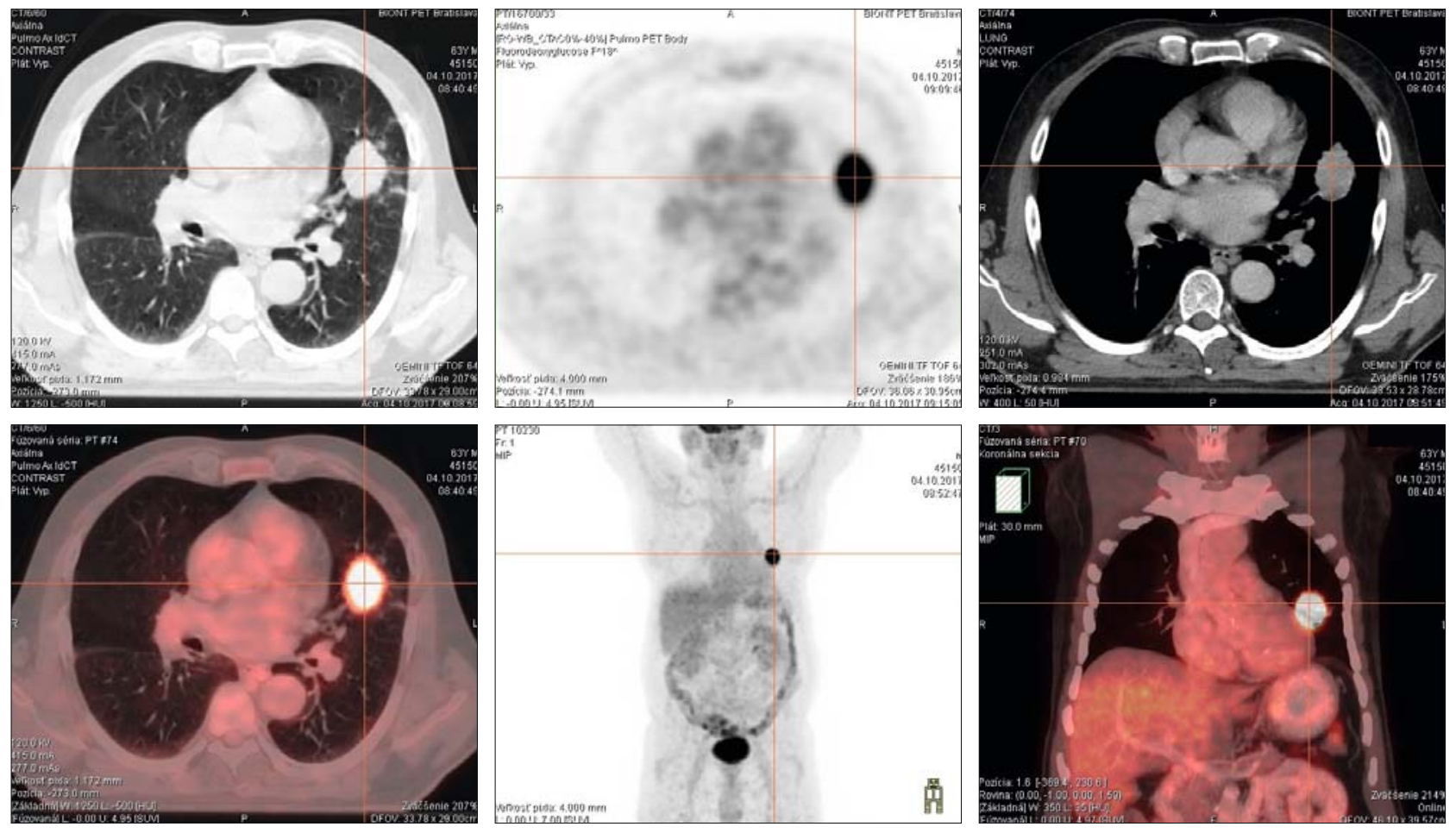

Fig. 1. PET/CT scan for radiotherapy planning (own material).

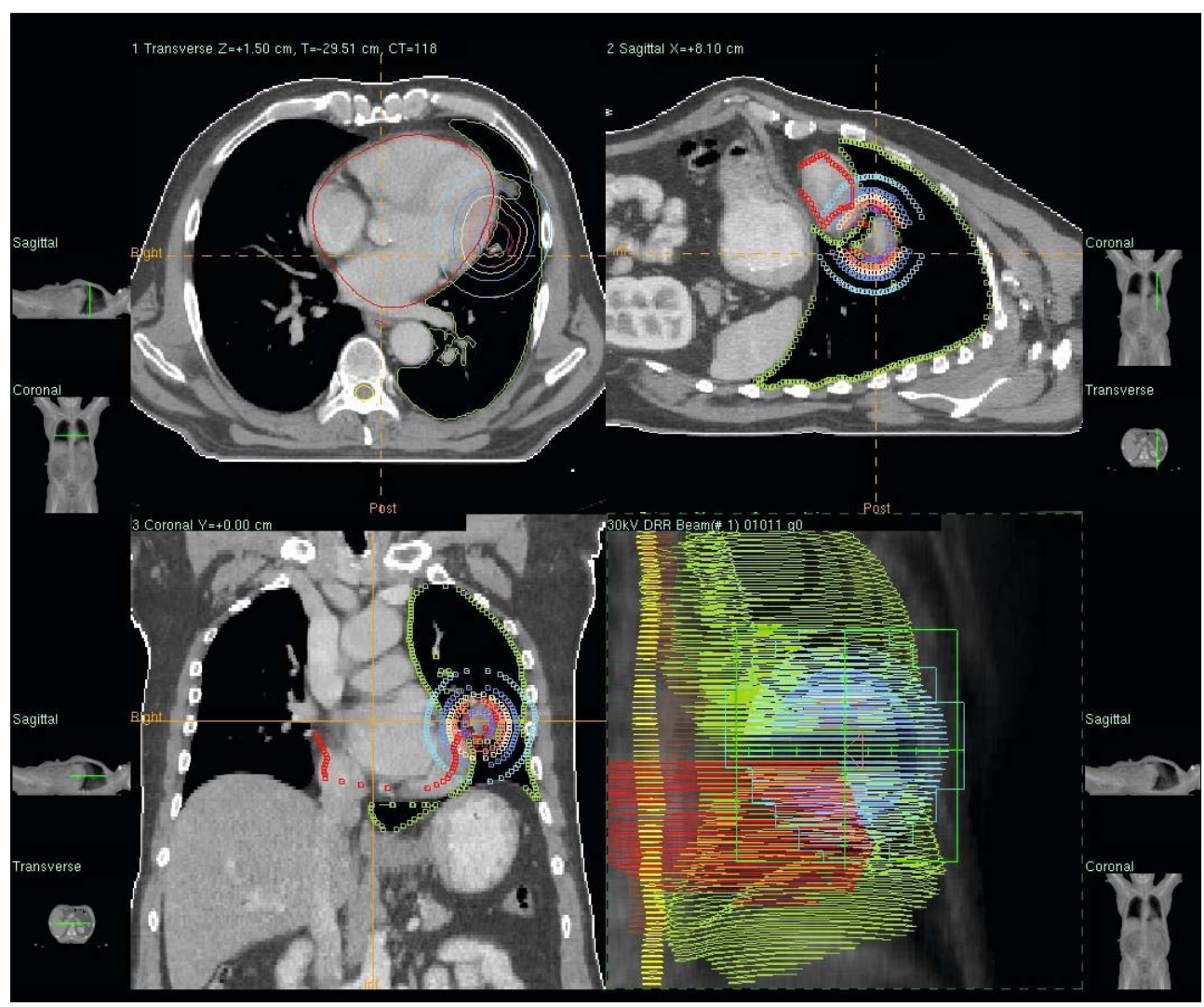

Fig. 2. Target volumes (own material). 
Tab. 1. Clinical characteristics of total 61 patients with advanced nonsmall cell lung cancer.

\begin{tabular}{lc}
\hline Patient characteristics & Data, $\mathrm{n}(\%)$ \\
\hline No. Of patients & 61 \\
\hline Age (years) & $27(44)$ \\
$\quad<65$ & $34(56)$ \\
$\geq 65$ & $13(21)$ \\
\hline Gender & $48(79)$ \\
$\quad$ Female & \\
$\quad$ Male & $17(28)$ \\
\hline Histology & $44(72)$ \\
AC & \\
SCC & $3(5)$ \\
TNM stage & $3(5)$ \\
IA & $5(8)$ \\
IIA & $22(36)$ \\
IIB & $28(46)$ \\
IIIA & \\
IIIB & $46(75)$ \\
Induction ChT & $15(25)$ \\
$\quad$ with & \\
without & $27(44)$ \\
Tumour volume (cc) & $34(56)$ \\
$<56$ & \\
$\geq 56$ & $28(46)$ \\
\hline Dose of RT (Gy) & $33(54)$ \\
$<61$ & $27(44)$ \\
$\geq 61$ & $34(56)$ \\
\hline SUVmax of primary tumour & $30(49)$ \\
$<11.4$ & $31(51)$ \\
$\geq 11.4$ & - tumour-node-metastasis \\
\hline SUVmax of lymphnodes & \\
$<3.12$ & \\
$\geq 3.12$ & \\
\hline AC-adenocarcinoma SCC-squmous & \\
\hline
\end{tabular}

tion chemotherapy $(\mathrm{ChT})$ followed by a definitive radiotherapy (RT) between January 2011 and December 2016. Inclusion criteria were: (I) histologically diagnosed NSCLC; (II) radiotherapy planned with 18F-FDG PET/CT; (III) stage IIIA and IIIB, including those in stage IA-IIB, but who were unable to undergo surgery or did not accept the operation; (IV) no history or concurrent diagnosis of another type of cancer; (V) overall survival (OS) more than 3 months.

The characteristics of the 61 patients are listed in Table 1, with the mean age of 65 years (range: 49-89). There were 13 women and 48 men. Fortyfour patients had squamous cell carcinoma and 17 patients had adenocarcinoma. The number of patients in the initial stage IIIA, IIIB and IA-IIB (who were unable to undergo surgery or did not accept the operation) was 22,28 and 11, respectively.

Fortysix patients received platinum-based chemotherapy according to the tumour histology. After four courses of the induction chemotherapy, all patients had PET/CT scan for radiotherapy planning (Fig. 1). However, none of our patients underwent PET/ CT for staging before ChT. The gross tumour volume (GTV) comprised the primary tumour and metabolically-positive lymphnodes on PET/CT and 27 patients had GTV smaller than 56cc compared to 34 patients with GTV greater than $56 \mathrm{cc}$ after the induction $\mathrm{ChT}$ (Tab. 1). The clinical target volume (CTV) included the GTV with

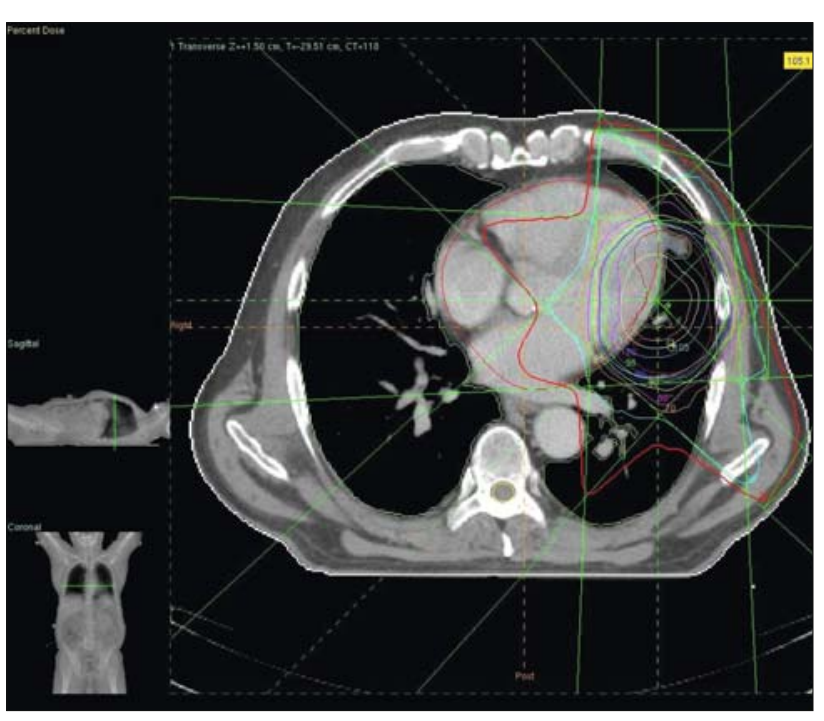

Fig. 3. Dose distribution (own material).

a 6-8 $\mathrm{mm}$ margin and the involved mediastinal lymphnode stations. Lymphnodes were irradiated to the same dose as the primary tumour. The CTV with a $10 \mathrm{~mm}$ margin was defined as the planning target volume (PTV) (Fig. 2). Radiotherapy was performed according to the PET/CT simulation with 6 or 18 -MV X-rays. The planned radiation dose was $40-70 \mathrm{~Gy}$, given in 2-2.5 Gy fractions over three and a half or seven weeks (Fig. 3).

All PET/CT studies were performed using a Philips Gemini TF 64 scanner after at least $6 \mathrm{~h}$ of fasting and $60 \mathrm{~min}$ after the intravenous injection of $370-740 \mathrm{MBq}$ of $18 \mathrm{~F}-\mathrm{FDG}$, depending on the patient's weight $(7.5 \mathrm{MBq} / \mathrm{kg})$. CT images were acquired from the thigh to the head with a 64 slice helical CT scanner and with the administration of i.v. contrast agents. PET images were then acquired over the same anatomical area after FDG administration, with the imaging time of $1.5 \mathrm{~min}$ ( $2 \mathrm{~min}$ for patients over $100 \mathrm{~kg}$ ) per bed position. CT based attenuation-corrected PET images were reconstructed using RAMLA (row-action maximum likelihood algorithm). Abnormal FDG uptake was defined as the uptake rate higher than the background activity in the surrounding tissue, and the FDG uptake intensity was quantified by calculating the standard uptake value (SUV). The SUV was calculated from the amount of FDG injected, the total body weight, and tissue uptake in the attenuation-corrected regional images as follows: SUV = (activity/unit volume) / (injected dose / total body weight). The SUVmax was defined as the peak SUV of the pixels with the highest counts within the region of interest (Fig. 4).

After RT completion, CT was performed within one month, and regular follow-ups were scheduled at 3-month intervals with alternating chest $\mathrm{CT}$ and PET/CT scans up to two years after RT and at 4-6-month intervals for the following three years. The median follow- up duration for these patients was 14 months (range: 6-58 months). Local control was assessed using a combination of clinical assessments, CT, PET/CT, or pathology. Local failure was defined as an increase in radiological abnormalities on CT or a serial escalation of the SUVmax on PET/CT within the RT field 

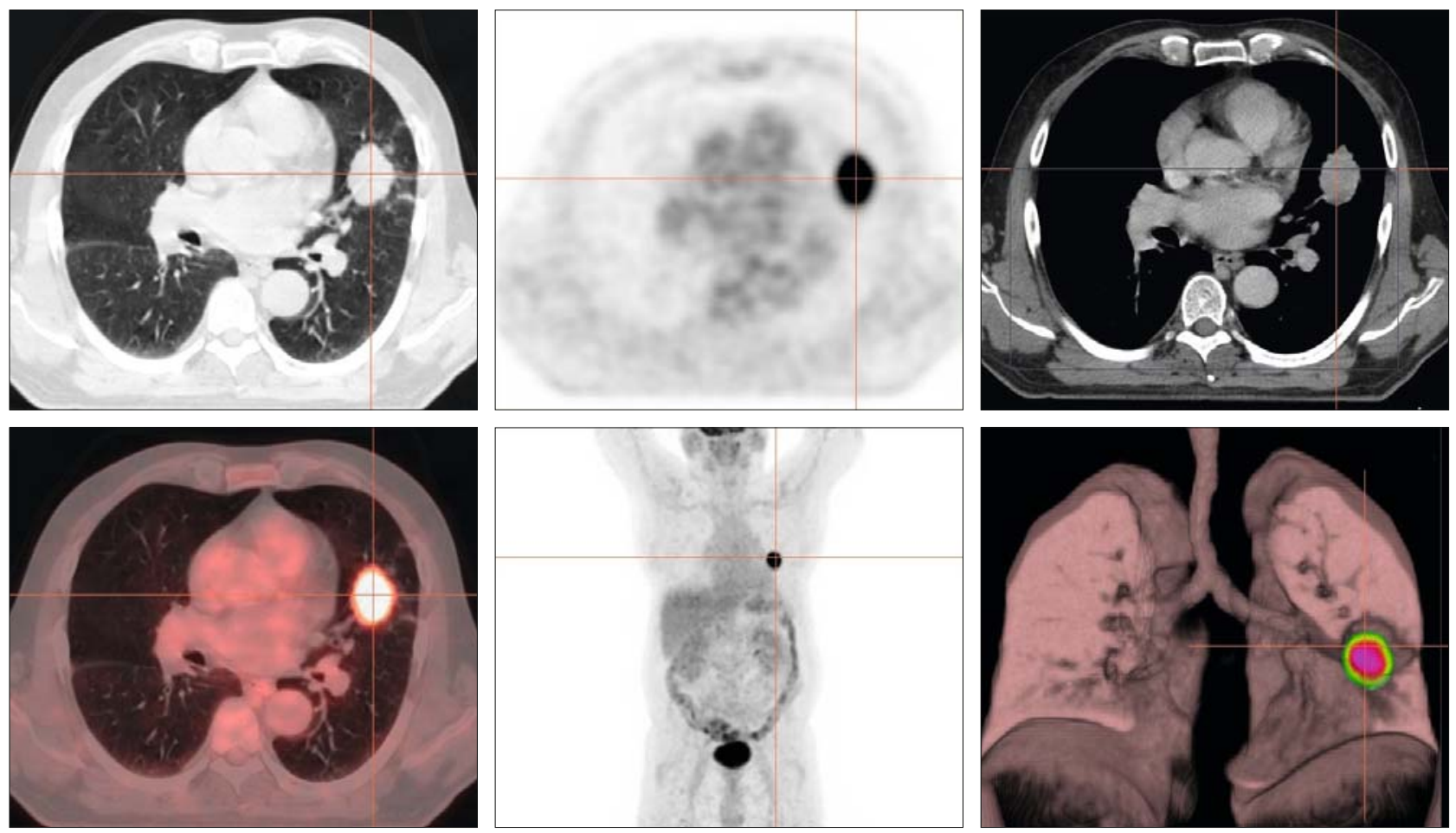

Fig. 4. The SUVmax within the region of interest (own material).

that was not judged to be radiation-induced pneumonitis. The overall survival was defined as the elapsed time from the RT end date to the date of death from any cause or the last follow-up date.

\section{Statistical analysis}

Survival probabilities were estimated using the Life tables method. The significance of the difference between the groups with respect to the studied parameters was assessed using the Fisher Exact test.

\section{Results}

We assessed SUVmax to analyse the association with local tumour control and survival. The mean SUVmax of primary tumour was 11.4 (range: $0.87-36.14$ ) and 3.12 of lymphnodes (range: 0 - 16.5) (Tab. 1). The value 11.4 of SUVmax for primary tumour and 3.12 for lymphnodes have been determined as the cut-off values because they represented intermediate values of SUVmax. For the minimum SUVmax values of primary tumour, which were 0.87 , and SUVmax of lymph nodes that were 0 , and which were recorded after induction $\mathrm{ChT}$, we assumed that it has occurred in the patients, who had a good response to the ChT. However, it was just an assumption, because we did not have the initial PET/CT for staging to compare and prove this claim.

Thirtysix per cent $(n=22)$ of the patients experienced a complete response $(C R)$ and twenty percent $(n=12)$ had a partial tumour response (PR) with the median survival of 13 months. Fortyfour per cent $(n=27)$ of the patients suffered from a progressive

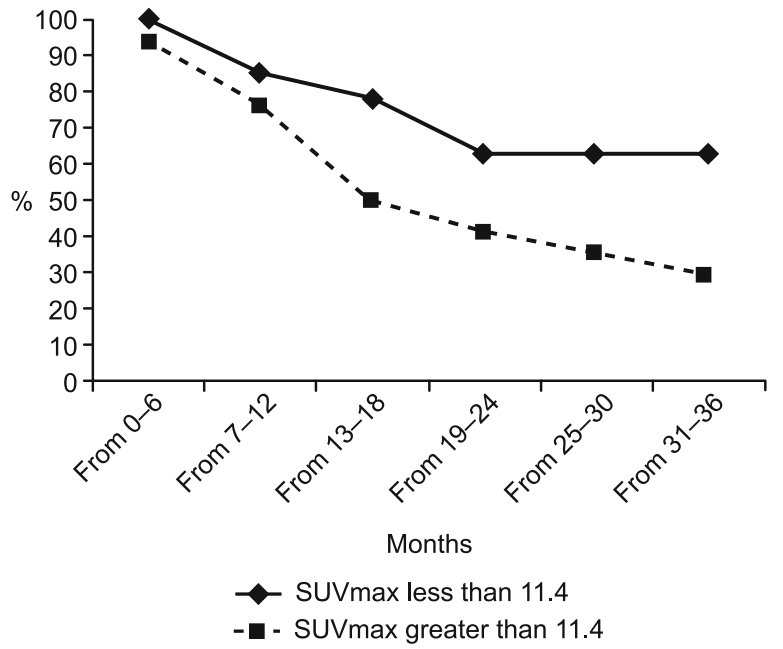

Fig. 5. The impact of SUVmax on 3-years OS.

disease with 10 months of median survival. Table 2 provide data on clinical response and survival for the whole group of patients.

There was a statistically significant difference between the two groups in the terms of clinical response and SUVmax $(<11.4$ versus $\geq 11.4)(p=0.0001)$ as well as for the survival of our patients and SUVmax $(p=0.0109)$. Patients, who had SUVmax lower than 11.4, had a median survival of 14 months (range: 6-58 months), and those patients with SUVmax higher than 11.4, had median survival of 10 months (range: 6-36 months). 
Tab. 2. Clinical response and patient survival.

\begin{tabular}{lc}
\hline Characteristics & Data, $\mathrm{n}(\%)$ \\
\hline Clinical response & \\
DCR & $34(56)$ \\
PD & $27(44)$ \\
\hline
\end{tabular}

PFS (median, months) $7.5(\min =6, \max =36)$

Survival (median, months) $14(\min =6, \max =58)$

$\overline{\mathrm{DCR}}$ - disease control rate (CR + PR), PD - progressive disease, PFS - progression free survival

Tab. 3. The ratio of SUVmax to GTV.

\begin{tabular}{llll}
\hline Variables & \multicolumn{2}{c}{ GTV $(\mathrm{cc})$} & $\mathrm{p}$ \\
\cline { 2 - 3 } & \multicolumn{1}{c}{$<56$} & $\geq 56$ & \\
\hline $\begin{array}{l}\text { SUVmax of primary tumour } \\
\quad<11.4\end{array}$ & $\begin{array}{l}17 \text { patients } \\
\quad 10 \text { patients }\end{array}$ & \multirow{2}{*}{0.0109} \\
$\geq 11.4$ & 10 patients & 24 patients & \\
\hline $\begin{array}{l}\text { SUV max of lymphnodes } \\
<3.12\end{array}$ & 20 patients & 10 patients & 0.0105 \\
$\geq 3.12$ & 10 patients & 21 patients & \\
\hline
\end{tabular}

Also, we analysed the ratio of SUVmax to GTV and it is shown in Table 3. Statistical correlation was observed between the tumour volume and SUVmax $(p=0.0109)$ and between lymphnodes status and SUVmax $(\mathrm{p}=0.0105)$. The median volume of the primary tumour was $56 \mathrm{cc}$ (range: $2-251 \mathrm{cc}$ ), and the mean SUVmax of tumours $\geq 56 \mathrm{cc}$ was statistically higher than those $<56 \mathrm{cc}$ as well as for SUVmax of lymph nodes (mean SUVmax $<3.12$ versus $\geq 3.12$ ). Among patients with SUVmax smaller than 11.4, seventeen patients lived and ten died, however, among patients with SUV max higher than 11.4, ten patients lived and twentyfour died.

Three years OS of patients with the SUVmax lower than 11.4 was $63 \%$ and $29 \%$ in patients with SUVmax higher than 11.4 (p $<0.0001$ ) (Fig. 5).

\section{Discussion}

PET/CT is a tool to gain biological information from tumours and the various PET/CT parameters associated with clinical outcomes in patients with NSCLC, with inconsistent results (12). To quantify a lesion on PET, the most commonly used parameter is the SUVmax, which reflects the voxel with the highest radioactivity concentration. The proposed mechanism was that poor prognosis was associated with the overexpression of glucose transporters and upregulation of hexokinase enzyme activity as reflected by the increased accumulation of FDG in malignant tumours $(13,14)$. Primary reduction ratio of the SUVmax (SRR), which means the change in the SUVmax of the primary tumour, has been studied as a prognostic or predictive factor and has a near-linear relationship with the percentage of non-viable tumour cells (15).

Chemoradiotherapy (ChRT) has been proven as the treatment of choice for patients with an advanced stage III NSCLC. However, some patients did not benefit from ChRT, and a high percentage of patients experienced a disease progression within the radiation field. Most of our patients had an induction ChT before RT. However, initially, all of them had CT scan for staging, not PET/CT. Despite that, the assumption of our study was that SUVmax of the planning PET/CT might be suggestive of an association between higher post-treatment SUV values and worse survival of patients with locally advanced NSCLC.

The prognostic value of FDG-PET after induction treatment has not been studied as thoroughly as at diagnosis. There is, however, increasing interest in determining the prognostic value of the post-therapy FDG-PET. Several studies have shown promising results, indicating that the post-therapy FDG-PET also has an independent prognostic value and is superior to that of CT (16).

Our study has also shown that the post-therapy 18F-FDG $\mathrm{PET} / \mathrm{CT}$ had a prognostic importance for patients with inoperable NSCLC. It has been shown that the degree of FDG uptake had a prognostic value after induction ChT.

Whether or not a pathologic complete response could be achieved is predictive for a more or less favourable outcome. Therefore, the pathologic complete response rate is an important endpoint for studies that evaluate new induction treatments (17).

Among our patients, there was a statistically significant difference between the two groups in terms of clinical response and SUVmax $(p=0.0001)$ as well as survival and SUVmax ( $\mathrm{p}=$ 0.0109). PET response was correlated with pathologic response, as well as the patient' survival.

Univariate analyses performed to determine a cut-off point for the SUV in the primary tumour to discriminate between a more or less favourable prognosis has ranged widely from 5 to 20. Vansteenkiste and Higashi etal showed that dichotomization with a broad range of SUVs gave significantly discriminative logrank probability values. This implies that the relationship between SUV and prognosis could be a gradual one rather than based on a threshold. It seems reasonable to hypothesize that there is no true cut-off point but, rather, a transition zone, within which the prognosis gradually worsens $(17,18)$.

In our study, SUVmax of the primary tumour with value of 11.4 has been determined as the cut-off value, which was represented by the mean value of heterogeneous data and likelihood of lymph node metastasis increases with an increase of the SUVmax of a primary lung cancer.

A tumour did not always have a uniform shape and a homogeneous composition, so tumour diameter could not represent the real tumour burden. Stiles etal provided the evidence in his study that SUVmax/tumour size was a stronger independent predictor of survival than SUVmax alone. However, all the patients enrolled in that study were at an early stage (IA-IIIA) $(6,7,8)$.

Although the association between SUVmax or SUVmax/tumour size and therapy response or survival in advanced NSCLC patients is still unclear, we analysed the influence of SUVmax and tumour volume on therapy response and survival. Furthermore, tumour size was associated with differences in SUV parameters. The values were increased for larger tumours. Therefore, our study showed a good correlation between tumour volume and SUVmax.

This study has demonstrated that in patients treated with an induction ChT followed by radical RT for inoperable NSCLC, higher SUVmax derived from a planning FDG-PET/CT scan significantly correlated with worse local control and poorer survival. 
133-138

\section{Conclusion}

In conclusion, we found a correlation between the maximum standardized uptake value and patient prognosis and lung cancer aggressiveness. Fluorodeoxyglucose maximum standardized uptake value was an important prognostic factor for local control and survival in 61 irradiated inoperable NSCLC patients.

\section{References}

1. Paesmans M. Prognostic and predictive factors for lung cancer. Breathe 2012; 9 (2): 113-122.

2. Berghmans T, Dusart M, Paesmans M, Hossein-Foucher C, Buvat I et al. Primary Tumor Standardized Uptake Value (SUVmax) Measured on Fluorodeoxyglucose Positron Emission Tomography (FDG-PET) is of Prognostic Value for Survival in Non-small Cell Lung Cancer (NSCLC). A Systematic Review and Meta-Analysis (MA) by the European Lung Cancer Working Party for the IASLC Lung Cancer Staging Project. J Thorac Oncol 2008; 3: 6-12.

3. Kinahan PE, Fletcher JW. PET/CT Standardized Uptake Values (SUVs) in Clinical Practice and Assessing Response to Therapy. Semin Ultrasound CT MR 2010; 31 (6): 496-505.

4. Shirai K, Abe T, Saitoh JI, Mizukami T, Irie D et al. Maximum standardized uptake value on FDG-PET predicts survival in stage I non-small cell lung cancer following carbon ion radiotherapy. Oncol Lett 2017; 13 : 4420-4426.

5. Machtay M, Duan F, Siegel BA, Snyder BS, Gorelick JJ et al. Prediction of survival by [18F]fluorodeoxyglucose positron emission tomography in patients with locally advanced non-small-cell lung cancer undergoing definitive chemoradiation therapy: results of the ACRIN 6668/RTOG 0235 trial. J Clin Oncol 2013; 31: 3823-3830.

6. Chen F, Yao Y, Ma C, Ma X, Wang Z et al. Ratio of maximum standardized uptake value to primary tumor size is a prognostic factor in patients with advanced non-small cell lung cancer. Transl Lung Cancer Res 2015; 4 (1): 18-26.

7. Stiles BM, Nasar A, Mirza F, Paul S, Lee PC et al. Ratio of Positron Emission Tomography Uptake to Tumor Size in Surgically Resected NonSmall Cell Lung Cancer. Ann Thorac Surg 2013; 95: 397-404.
8. Alshuhayeb A., Gilbert S, Seely AJE, Shamji FM, Sundaresan S. Prognostic Significance of Standardized Uptake Value of PET Scan in Non-Small Cell Lung Cancer. J Cancer Ther 2016; 7: 186-196.

9. Duan XY, Wang W, Li M, Li Y, Guo YM. Predictive significance of standardized uptake value parameters of FDG-PET in patients with nonsmall cell lung carcinoma. Brazil J Med Biol Res 2015; 48 (3): 267-272.

10. Bryant AS, Cerfolio RJ, Klemm KM, Ojha B. Maximum standard uptake value of mediastinal lymph nodes on integrated FDG-PET-CT predicts pathology in patients with non-small cell lung cancer. Ann Thorac Surg 2006; 82: 417-422.

11. Huang W, Zhou T, Ma L, Sun H, Gong H et al. Standard uptake value and metabolic tumor volume of $18 \mathrm{~F}-\mathrm{FDG}$ PET/CT predict shortterm outcome early in the course of chemoradiotherapy in advanced nonsmall cell lung cancer. Eur J Nucl Med Mol Imaging 2011; 38: 1628-1635.

12. Vaidya M, Creach KM, Frye J, Dehdashti F, Bradley JD, El Naqa I. Combined PET/CT image characteristics for radiotherapy tumor response in lung cancer. Radiother Oncol 2012; 102: 239-245.

13. Jin F, Zhu H, Fu Z, Kong L, Yu J. Prognostic value of the standardized uptake value maximum change calculated by dual-time-point $18 \mathrm{~F}$-fluorodeoxyglucose positron emission tomography imaging in patients with advanced non-small-cell lung cancer. OncoTargets Ther 2016; 9: 2993-2999.

14. Lin MY, Wu M, Brennan S, Campeau MP, Binns DS et al. Absence of a Relationship between Tumor 18F-fluorodeoxyglucose Standardized Uptake Value and Survival in Patients Treated with Definitive Radiotherapy for Non-Small-Cell Lung Cancer. J Thorac Oncol 2014; 9 (3): 377-382.

15. Jeong JU, Chung WK, Nam TK, Song JY, Yoon MS et al. Early Metabolic Response on 18F-Fluorodeoxyglucose-Positron-emission Tomography/Computed Tomography After Concurrent Chemoradiotherapy for Advanced Stage III Non-small Cell Lung Cancer Is Correlated with Local Tumor Control and Survival. Anticancer Res 2014; 34: 2517-2524.

16. de Geus-Oei LF, van der Heijden HFM, Corstens FHM, Oyen WJG. Predictive and Prognostic Value of FDG-PET in Nonsmall-Cell Lung Cancer. Cancer 2007; 110 (8): 1654-1664.

17. de Geus-Oei LF, Oyen WJG. Predictive and prognostic value of FDG-PET. Cancer Imaging 2008; 8: 70-80.

18. Shimizu K, Okita R, Saisho S, Yukawa T, Maeda A. Clinical significance of dual-time-point 18F-FDG PET imaging in resectable non-small cell lung cancer. Ann Nucl Med 2015; 29: 854-860. 